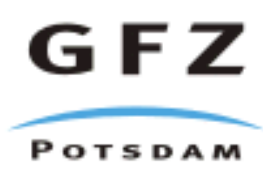

Originally published as:

Tsoulis, D., Petrovic, S., Kilian, N. (2008): Theoretical and Numerical Aspects of the Geodetic Method for Determining the Atmospheric Refraction Coefficient Using Simultaneous and Mutual Zenith Observations. - Journal of Surveying Engineering, 134, 1, 312 ,

DOI: 10.1061/(ASCE)0733-9453(2008)134:1(3). 


\title{
Theoretical and numerical aspects of the geodetic method for determining the atmospheric refraction coefficient using simultaneous and mutual zenith observations
}

\author{
Dimitrios Tsoulis ${ }^{1}$, Svetozar Petrović ${ }^{2}$, Nicole Kilian ${ }^{3}$ \\ ${ }^{1}$ Department of Geodesy and Surveying \\ Aristotle University of Thessaloniki \\ 54124 Thessaloniki, Greece \\ email: tsoulis@topo.auth.gr \\ ${ }^{2}$ Department 1: Geodesy and Remote Sensing \\ GeoForschungsZentrum Potsdam \\ Telegrafenberg C 3, 14473 Potsdam, Germany \\ email: sp@gfz-potsdam.de \\ ${ }^{3}$ Gubener Str. 39, 10243 Berlin, Germany \\ email: nekn@gmx.de
}

Manuscript submitted to

Journal of Surveying Engineering

2nd revised manuscript version of September 6, 2007 


\begin{abstract}
The estimation of the atmospheric refraction coefficient by conducting simultaneous and mutual zenith observations is a well-known geodetic procedure. The respective mathematical model is based on certain assumptions regarding the actual path followed by the optical ray while propagating in the atmosphere (spherical symmetric model). Starting from this model an approximate solution can be derived, which is generally accepted in the literature on the subject. The present paper examines the mathematical model of this geodetic technique in a rigorous manner, which results in an exact solution for the computation of the atmospheric refraction coefficient or equivalent for the zenith angle correction that is due to the effect of the atmospheric refraction. Since the direct computation of the exact solution is rather complicated, an iterative way of computing the same quantity is derived, which can be realized easily on any pocket calculator. In order to assess the derived formulae we investigate the applicability of the exact, the convergence rate of the iterative and the errors introduced by the approximate formulae, by conducting a representative series of synthetic numerical experiments. The overall discussion aims to provide a helpful insight into the geometric and theoretical features of this traditional geodetic method. To practitioners that attempt to apply the standard correction formulae to zenith observations in order to compensate the effect of atmospheric refraction in the frame of typical surveying applications, such as trigonometric leveling, the paper also points out possible numerical errors introduced by the traditional approximate formulae.
\end{abstract}

Keywords (according to ASCE Thesaurus): Leveling, Refraction, Height, Surveys 


\section{Introduction}

The study of the effect of atmospheric refraction to the travel path of optical rays has occupied a prominent position in the research of optics and related applied disciplines from the very early days. The first historical reference to the concepts of reflection and refraction is assigned apparently to Euclid, if we follow an anonymous Latin translation of the 13th century A.D. of one of Euclid's works with the general title Catoptrica (Murdoch 1984). A more detailed description of refraction has been undertaken by the English philosopher Roger Bacon (1214-1294), who related the optical deformation of the image of an object with the characteristics, i.e. optical density, of the respective medium in which the light propagation is performed. Finally, renaissance and the dawn of the 17th century A.D. witnessed the rigorous mathematical formulation of the refraction law accompanied with an interesting historical anecdote. The law was defined by Willebrord Snell (1580-1626) prior to its official publication a couple of years later by the French philosopher and mathematician Rene Descartes (1596-1650) in his work Dioptrics, published in 1637 (Cohen, 1980).

The term atmospheric or geodetic refraction, is found in the relevant surveying literature as a means to describe the alteration in the direction of the light curve as it propagates through the different layers of the lower part of the Earth's atmosphere in the frame of standard surveying applications. Primary observed quantities of surveying engineering, (directions, angles, distances) are obtained by observing either a reflected light beam from a target located in the field towards the measuring device (passive light receivers, such as theodolites or levels) or by studying the propagation of a transmitted electromagnetic signal in the atmosphere from the instrument to an appropriately selected target and backwards (electromagnetic distance measurement). Therefore, it is of fundamental interest to have the best possible knowledge of the medium characteristics (optical density of the atmosphere and its gradient) at the specific time and place and theoretically for every point along the respective profile. When such a rigorous analysis is not possible, it is equally important (and in most cases sufficient) to have a rough estimate of the order of magnitude and sign of the atmospheric refraction coefficient as well as its effect to observed quantities (zenith angle observations, leveling rod readings etc). The latter assumes a user defined light propagation model for the area of interest, that can represent the real medium structure only approximately, thus offering an estimate of the respective corrections. However, even these approximate solutions for the atmospheric refraction often are presented in a truncated generalized manner in the respective literature, not corresponding rigorously to the applied geometric propagation model. This means, adopting a rather simple geometric light propagation model might be necessary if there is no better possibility. However, there is no need to avoid the rigorous solution in the frame of the accepted model by making further approximations, which were computationally profitable in the age of logarithmic and trigonometric tables. Unfortunately this approach still dominates the respective literature. 
In this paper, we are going to investigate the main features of the traditional geodetic method of atmospheric refraction evaluation using the simultaneous mutual zenith observations from two field points. We are going to comment both on the theoretical as well as on the numerical aspects of the respective formulae found in several widely cited geodetic references and textbooks. The goal is to provide a meaningful mathematical description of the postulated physical and geometric model, by pointing out at the same time the limits of using some of the expressions presented here in real-life applications, e.g., in order to provide some decent corrections to trigonometric or geometric leveling field data.

Traditional geodetic refraction modeling incorporates a spherical symmetric model for the propagation of light curves through the atmospheric medium (Jordan et al 1956, Rueger 1996, Kahmen and Faig 1988). There have been several investigations of the possibilities to determine local refraction coefficients in small regions by measuring temperature gradients (see, e.g., Gottwald 1985). Current applications for the atmospheric refraction determination involve active experimental setups including digital imaging sensors and highly efficient instruments for the field measurement of different atmospheric turbulence parameters and the inclusion of these data into rigorous optical turbulent models (a small excerpt of a relatively wide relevant scientific activity over the last decade is given by Flach and Maas 1999, Hennes 2002, Witte and Binnenbruck 2000).

The combination of the mathematical rigor of the available turbulence models with the precision and efficiency of elaborate instruments (scintillometers, digital cameras, video-theodolites etc), permits nowadays an extremely advanced means of atmospheric parameter modeling, including the determination of the atmospheric refraction coefficient or index. However, due to the high cost of the respective equipment and the a-priori restrictions of the applicability of the method to a certain range of distances, these techniques still have rather an experimental character (Flach and Maas 1999, Witte and Binnenbruck 2000). On the other hand the knowledge of the behavior of the atmospheric refraction over a selected field area is important to the practitioner who is to perform typical surveying applications, such as geometric or trigonometric leveling and wishes to calculate the effect of atmospheric refraction on his primary observations, e.g. leveling rod readings or zenith angle observations. The quantification of the effect of atmospheric refraction to the aforementioned quantities may be realized only if a certain model for the propagation of light in the specific medium (region of the atmosphere where measurements take place) is adopted, based on either real scintillometric data that have been obtained for the area or some approximate theoretical model that can be described analytically. The examination of the latter possibility is the topic of the present paper. By examining the theoretical and numerical aspects of the traditional method of evaluating the atmospheric refraction coefficient by using the mutual zenith observations that were obtained from two different field points from simultaneous observations, both a geometric as well as a numerical insight into the nature of the specific technique is obtained.

After the mathematical formulation of the problem, when a spherical symmetric light propagation model 
is adopted, three different solution strategies are presented that lead to an iterative, an algebraic and to the usual approximate solution (found in standard geodetic references) for the computation of the atmospheric refraction coefficient. The numerical investigation with simulated data allows a helpful numerical assessment of the approximate formula especially in the frame of its practical use in trigonometric leveling.

\section{Mathematical model}

The geometrical features of the geodetic method of estimating the atmospheric refraction coefficient using simultaneous and mutual zenith measurements are shown in Figure 1. At the instrument points $A$ and $B$ (these are rigorously defined as the intersections between the vertical and the optical theodolite axes at the two locations, not to be interpreted as the respective field points) the zenith observations $z_{A}^{\prime}$ and $z_{B}^{\prime}$ are carried out respectively under the model assumption that a spherical symmetric model describes the effect of the atmospheric refraction. Thus, the deviation of the real optical ray path from the theoretical linear line of sight connecting $A$ with $B$ is the same at the two locations while obeying a strict spherical symmetric model for the space between $A$ and $B$, which leads consequently to the same angle deviation $\delta$ of the observed zenith angles $z_{A}^{\prime}$ and $z_{B}^{\prime}$ with respect to the theoretically defined $z_{A}$ and $z_{B}$, in other words it holds

$$
z_{A}=z_{A}^{\prime}+\delta \quad, \quad z_{B}=z_{B}^{\prime}+\delta
$$

The problem of the computation of angle $\delta$ coincides with the problem of determining the curvature of the applied spherical ray propagation model which is implied in Figure 1. The latter is connected with the geodetic refraction coefficient $k$, by definition through the relation (Rüeger, 1996)

$$
k=\frac{R_{o}}{r}
$$

where $r$ denotes the radius of the spherical ray propagation model and $R_{o}$ the chosen constant which by convention equals the mean Earth radius.

In order to compute angle $\delta$ in Equation (1) exploiting the direct field measurements $z_{A}^{\prime}$ and $z_{B}^{\prime}$ we refer to the geometry of Figure 1. In triangle $A B C$, with $A C$ and $B C$ denoting the distances along the directions of the plumb line defined at points $A$ and $B$ respectively, and supposing that they intersect at point $C$, the following angle relation holds

$$
\alpha+\beta+\gamma=\pi
$$


If we add $2 \delta$ at both sides of this equation we obtain

$$
\begin{aligned}
\alpha+\beta+\gamma+2 \delta & =\pi+2 \delta \Longrightarrow \\
(\alpha+\delta)+(\beta+\delta)+\gamma & =\pi+2 \delta \Longrightarrow \\
\pi-z_{A}^{\prime}+\pi-z_{B}^{\prime}+\gamma & =\pi+2 \delta
\end{aligned}
$$

which leads to the following expression for quantity $\delta$

$$
\delta=\frac{1}{2}\left(\pi+\gamma-z_{A}^{\prime}-z_{B}^{\prime}\right)
$$

In the case that the angle $\gamma$ between the directions of the plumb line at $A$ and $B$ is known with an appropriate accuracy from another independent source, for example astronomical observations or local deflections of the vertical, then Equation (5) provides a direct means of computing the effect of refraction on the simultaneous zenith observations from $A$ to $B$ and vice versa. Having $z_{A}^{\prime}$ and $z_{B}^{\prime}$ as field measurements and angle $\delta$ computed from (5), one may compute angles $\alpha$ and $\beta$ of $A B C$ as

$$
\alpha=\pi-\left(z_{A}^{\prime}+\delta\right) \quad, \quad \beta=\pi-\left(z_{B}^{\prime}+\delta\right)
$$

In the same triangle one gets consequently for the slope distance $s=(A B)$ the following exact computational formula

$$
s=R \frac{\sin \gamma}{\sin \beta}=R \frac{\sin \gamma}{\sin \left(z_{A}-\gamma\right)}
$$

where $R=(A C)$ is defined on the plumb line direction through $A$ and describes geometrically the geometric distance between $A$ and the intersection of both plumb lines (at $A$ and $B$ respectively) at $C$ defined on the plumb line direction through $A$. At this point we should comment on quantities $R$ and $R_{o}$, especially with respect to the numerical implementation of $R$. Quantity $R_{o}$ in (2) represents a mean Earth radius, whereas parameter $R$ expresses a curvature radius of the equipotential surfaces in some spherical mean or local approximation. In other words, $R$ refers to the radius of the sphere, which for the specific region approximates optimally a spherical representation of the equipotential surface defined at $A$ in the frame of a spherical approximation of the Earth's gravity field. The two plumb lines defined at points $A$ and $C$ respectively do not actually intersect, as they belong to different vertical planes. However 
it is of negligible error to consider the projections of both plumb lines to a common vertical plane, chosen as close as possible to the previous two, where point $C$ can be defined. In this way Figure 1 can be obtained. An important question which arises now, is what numerical value for $R$ should one use in any possible numerical considerations, where parameter $R$ is entering. It may be shown, by performing a detailed error propagation study, that the accuracy with which angle $\delta$ is obtained is substantially more sensitive to errors in quantity $\gamma$ than the accuracy with which radius $R$ is given. From formula (5) it is visible that the error in $\gamma$ enters into $\delta$ directly with a factor of 0.5 . The small influence of the errors in $R$ can be roughly estimated from equation (13). In other words, small errors in the knowledge of the actual plumb line directions have a much greater impact on the accuracy of the computed $\delta$ values than big errors in $R$. Adding to this consideration the fact that, at least near the Earth's surface, the curvature of the equipotential surfaces is of the same order of magnitude as $R_{o}$, we may use some given numerical value for $R_{o}$ as a fairly good numerical approximation for $R$, i.e. without performing a noticeable or detectable error. Thus, performing a permissible and suitable approximation we will use in the sequel some admissible mean Earth radius value, when performing numerical calculations which involve $R$.

Taking further into account the spherical ray propagation model which is presented in detail in Figure 2, we obtain for quantity $\delta$ the expression

$$
\sin \delta=\frac{s / 2}{r} \Longrightarrow \quad r=\frac{s}{2 \sin \delta}
$$

and finally from Equation (2) we obtain for the refraction coefficient

$$
k=\frac{R_{o}}{r}=\frac{2 R_{o} \sin \delta}{s}
$$

\section{Iterative solution}

Since it is merely an exception that angle $\gamma$ is a given quantity, the computation of angle $\delta$ expressing the effect of a spherical symmetric atmospheric refraction model onto the observed zenith angles cannot be usually computed from equation (5) directly, but involves some solution strategy for the determination of the unknown angle $\gamma$. The present section deals with an iterative computation of $\gamma$. An algebraic approach is presented in the following section.

Let us assume, that apart from the mutual zenith angle observations $z_{A}^{\prime}$ and $z_{B}^{\prime}$, the slope distance $s$ between the two field points $A$ and $B$ and the radius $R$ (see Figure 1) are known parameters as well. At this point having only this information equation (5) cannot be evaluated lacking the knowledge of angle 
$\gamma$. However, from triangle $A B C$ and relation (5) it follows for angle $\beta$

$$
\beta=\pi-z_{B}^{\prime}-\delta=\frac{1}{2}\left(\pi-\gamma+z_{A}^{\prime}-z_{B}^{\prime}\right)
$$

The law of sines for the same triangle gives

$$
\frac{\sin \gamma}{s}=\frac{\sin \beta}{R}
$$

From equations (10) and (11) we obtain the following trigonometric equation for the computation of $\gamma$

$$
\sin \gamma=\frac{s}{R} \sin \left(\frac{1}{2}\left(\pi-\gamma+z_{A}^{\prime}-z_{B}^{\prime}\right)\right)
$$

Equation (12) is a non-linear equation with respect to the unknown parameter $\gamma$, which also enters both sides of the equation. This implies that an iterative solution is possible, however the iteration scheme has to be investigated with respect to its convergence. If the iteration converges then the obtained solution may be regarded equivalent to the respective exact solution for $\gamma$.

For the iterative solution of non-linear equations there exist different iterative schemes (see e.g. Bronstein and Semendjajew 2002). The most commonly applied is the simple iterative scheme $x_{i+1}=f\left(x_{i}\right)$ that computes at the iteration step $(i+1)$ the parameter $x$ from its value in iteration step $i$. Fundamental prerequisite of the method is that the initial non-linear equation has to be rewritten in the form $x=f(x)$. In order to guarantee the convergence of the iteration procedure, function $f(x)$ has to fulfill certain conditions. More precisely, its first derivative with respect to $x$ has to exist and be continuous within a certain domain in the neighborhood of the solution $x$. Furthermore, the starting value of the iteration $x_{o}$ has to be chosen from this specific domain, while for the same domain the relation $\left|f^{\prime}(x)\right|<1$ has to be valid.

In order to solve for angle $\gamma$ the first step is to rewrite equation (12) in the desired iteration form $x_{i+1}=f\left(x_{i}\right)$. One obtains directly the expression

$$
\gamma_{i+1}=\arcsin \left(\frac{s}{R} \sin \left(\frac{1}{2}\left(\pi-\gamma_{i}+z_{A}^{\prime}-z_{B}^{\prime}\right)\right)\right)
$$

Using the abbreviation

$$
\varepsilon=\frac{1}{2}\left(\pi+z_{A}^{\prime}-z_{B}^{\prime}\right)
$$


(which is a given quantity) only the validity of the aforementioned convergence criteria for the function

$$
f(\gamma)=\arcsin \left(\frac{s}{R} \sin \left(\varepsilon-\frac{\gamma}{2}\right)\right)
$$

has to be investigated. The derivative of the last expression with respect to parameter $\gamma$ is

$$
f^{\prime}(\gamma)=\frac{1}{\sqrt{1-\frac{s^{2}}{R^{2}} \sin ^{2}\left(\varepsilon-\frac{\gamma}{2}\right)}}\left(-\frac{1}{2} \frac{s}{R} \cos \left(\varepsilon-\frac{\gamma}{2}\right)\right)
$$

or equally

$$
f^{\prime}(\gamma)=-\frac{s \cos \left(\varepsilon-\frac{\gamma}{2}\right)}{2 R \sqrt{1-\frac{s^{2}}{R^{2}} \sin ^{2}\left(\varepsilon-\frac{\gamma}{2}\right)}}
$$

Since $s^{2} / R^{2}<1$, the derivative (17) is both defined and continuous for any $\gamma \in \Re$, which means that the starting value $\gamma_{o}$ can be chosen arbitrarily, if we prove that the condition $\left|f^{\prime}(\gamma)\right|<1$ is valid. This second constraint will obviously hold only for some specific range of the involved parameter values.

Since the following inequality holds

$$
\frac{s^{2}}{R^{2}} \sin ^{2}\left(\varepsilon-\frac{\gamma}{2}\right) \leq \frac{s^{2}}{R^{2}}
$$

then the subsequent relation is valid

$$
1-\frac{s^{2}}{R^{2}} \sin ^{2}\left(\varepsilon-\frac{\gamma}{2}\right) \geq 1-\frac{s^{2}}{R^{2}}
$$

Consequently

$$
\frac{1}{\sqrt{1-\frac{s^{2}}{R^{2}} \sin ^{2}\left(\varepsilon-\frac{\gamma}{2}\right)}} \leq \frac{1}{\sqrt{1-\frac{s^{2}}{R^{2}}}}
$$

Combining equations (20) and (17) we get

$$
\left|f^{\prime}(\gamma)\right| \leq-\frac{s\left|\cos \left(\varepsilon-\frac{\gamma}{2}\right)\right|}{2 R \sqrt{1-\frac{s^{2}}{R^{2}}}} \leq \frac{s}{2 R \sqrt{1-\frac{s^{2}}{R^{2}}}}
$$


Thus the investigation of the validity of the condition $\left|f^{\prime}(\gamma)\right|<1$ is equivalent to examining the condition

$$
\frac{s^{2}}{4 R^{2}\left(1-\frac{s^{2}}{R^{2}}\right)}=\frac{s^{2}}{4 R^{2}-4 s^{2}}<1
$$

From the last inequality one obtains finally the equivalent condition

$$
s<\sqrt{\frac{4}{5}} R
$$

which obviously holds for any practically possible slope distance $s=(A B)$ between the two field points.

The above investigation has led to the conclusion that the iteration scheme (13) produces a convergent, thus exact solution for $\gamma$. The evaluation algorithm consists of a choice for the starting iteration value $\gamma_{o} \in \Re$, for example $\gamma_{o}=0$. Then the iteration for $\gamma$ follows equation (13), where the procedure is repeated until the envisaged accuracy level has been reached. This can be readily obtained by comparing the numerical values of $\gamma_{i+1}$ with $\gamma_{i}$ at the conclusion of every single iteration step.

The results of the iteration procedure are linked to the computations of parameters $\delta, k, z_{A}$ and $z_{B}$ also through an iterative algorithm. Thus, the correction $\delta_{i+1}$ to the observed zenith angles, the refraction coefficient $k_{i+1}$ and the corrected zenith angles $\left(z_{A}\right)_{i+1}$ and $\left(z_{B}\right)_{i+1}$, all referring to the iteration step $(i+1)$, can be computed respectively from the following scheme

$$
\begin{aligned}
& \delta_{i+1}=\frac{1}{2}\left(\pi+\gamma_{i+1}-z_{A}^{\prime}-z_{B}^{\prime}\right) \\
& k_{i+1}=\frac{2 R \sin \delta_{i+1}}{s} \\
& \left(z_{A}\right)_{i+1}=z_{A}^{\prime}+\delta_{i+1} \\
& \left(z_{B}\right)_{i+1}=z_{B}^{\prime}+\delta_{i+1}
\end{aligned}
$$

Important is at this point to comment on the convergence speed, i.e. to define the number of iterations that is necessary in order to obtain the desired accuracy level. The convergence speed of an iteration process can be discussed both theoretically as well as numerically. Although theory describes adequately the issue of convergence speed of a certain iteration procedure, some cases do exist, where the numerical application reveals 'non-predicted' convergence characteristics. In order to gain an insight into the numerical aspects 
of the specific iteration procedure we recall the standard representation of the iteration error $e_{i}=\left|\gamma-\gamma_{i}\right|$ referring to iteration step $i$ through the following approximative expression (Bronstein and Semendjajew 2002)

$$
e_{i} \approx\left|f^{\prime}(\gamma)\right|\left|e_{i-1}\right| \approx\left|f^{\prime}(\gamma)\right|^{i}\left|\gamma-\gamma_{o}\right|
$$

Recalling equation (21) and taking into account the fact that the method of mutual zenith observations cannot by applied to extremely large distances, i.e. by setting $s=100 \mathrm{~km}$ as the worse scenario and using for $R$ the numerical value $R=6400 \mathrm{~km}$, the following inequality for $\left|f^{\prime}(\gamma)\right|$ is obtained

$$
\left|f^{\prime}(\gamma)\right| \leq 0.0078
$$

Using $\gamma_{o}=0$ as the starting value for the iteration process we get $\left|\gamma-\gamma_{o}\right|=\gamma$ and for slope distances less than $100 \mathrm{~km}$ the realistic numerical restriction for angle $\gamma$, that $\gamma \leq 0.0156 \mathrm{rad}$. Inserting these numerical values into equation (28) we have

$$
e_{i} \leq 0.0078^{i} \times 0.0156 \mathrm{rad}
$$

Computing this quantity and for the first couple of iterations we obtain $e_{1} \leq 0.00012 \mathrm{rad}=7.7$ mgon $\left(24.94^{\prime \prime}\right), e_{2} \leq 0.000000949 \mathrm{rad}=0.06 \mathrm{mgon}\left(0.19^{\prime \prime}\right)$ and $e_{3} \leq 7.4 \times 10^{-9} \mathrm{rad}=0.47 \mu$ gon $\left(0.001^{\prime \prime}\right)$. Thus, after only three iterations the accuracy of the computed iterated angle $\gamma$ obtains numerical level that satisfy well any imaginable accuracy demands. For most real examples two iterations should be already sufficient. If we restrict ourselves to slope distances of up to $10 \mathrm{~km}$, we obtain for the derivative of function $f(\gamma)$, the angle $\gamma$ itself and the iteration error estimate the numerical values $\left|f^{\prime}(\gamma)\right| \leq 0.00078$, $\gamma \leq 0.00156 \mathrm{rad}$ and $e_{1} \leq 0.08$ mgon $\left(0.26^{\prime \prime}\right)$ respectively. For most of these cases $(s<10 \mathrm{~km})$ a single iteration seems to be adequate.

From the preceding investigations we can conclude that the iteration process for the computation of the 'geocentric' angle $\gamma$ between two field points is efficient and converges very quickly to an accuracy level that is sufficient for most real examples. The respective numerical computations are very simple, they can be carried out with any pocket calculator (disposing with trigonometric functions) and provide an interesting and useful detail (geocentric angle) of the applied spherical approximation model.

In order to obtain a more practical idea of the corresponding calculations for the case of a real example we examine the following scenario. Let $z_{A}^{\prime}=89.9870$ gon $\left(80^{\circ} 59^{\prime} 17.88^{\prime \prime}\right)$ and $z_{B}^{\prime}=110.0364$ gon $\left(99^{\circ}\right.$ $1^{\prime} 57.94^{\prime \prime}$ ) be the numerical values of two simultaneously observed zenith angles between field points $A$ and $B$ according to Figure 1. Furthermore, let the slope distance $s=(A B)=5 \mathrm{~km}$ and the numerical 
value for a mean Earth radius, also approximating in the frame of the present discussion the curvature radius of the equipotential surfaces in a local approximation, be set to $R=6370 \mathrm{~km}$. We commence the iteration procedure (13) with the starting value $\gamma_{o}=0$. After only two iteration steps we obtain for each iteration

$$
\begin{aligned}
& \gamma_{1}=49.352 \text { mgon }\left(2^{\prime} 39.9^{\prime \prime}\right) \Longrightarrow \delta_{1}=12.976 \operatorname{mgon}\left(42.04^{\prime \prime}\right) \\
& \gamma_{2}=49.349 \operatorname{mgon}\left(2^{\prime} 39.88^{\prime \prime}\right) \Longrightarrow \delta_{2}=12.974 \operatorname{mgon}\left(42.03^{\prime \prime}\right)
\end{aligned}
$$

which corresponds to an accuracy in the computation of angles $\gamma$ and $\delta$ of an order of magnitude $10^{-2}$ mgon and $10^{-3}$ mgon respectively. For the related quantities $k, z_{A}$ and $z_{B}$ the respective numerical results that are obtained at each iteration step are as follows

$$
\begin{aligned}
& k_{1}=0.519349, z_{A}^{1}=89.999975 \operatorname{gon}\left(80^{\circ} 59^{\prime} 59.919^{\prime \prime}\right), z_{B}^{1}=110.049376 \text { gon }\left(99^{\circ} 2^{\prime} 39.978^{\prime \prime}\right) \\
& k_{2}=0.519269, z_{A}^{2}=89.999974 \operatorname{gon}\left(80^{\circ} 59^{\prime} 59.916^{\prime \prime}\right), z_{B}^{2}=110.049374 \operatorname{gon}\left(99^{\circ} 2^{\prime} 39.972^{\prime \prime}\right)
\end{aligned}
$$

We note that the numerical values $k=0.52, z_{A}=90.0000$ gon $\left(81^{\circ}\right)$ and $z_{B}=110.0494$ gon $\left(99^{\circ} 2^{\prime}\right.$ $\left.40.06^{\prime \prime}\right)$ used for the construction of the example can be possibly obtained already from the first iteration step with a satisfactory accuracy.

The magnitude of neglecting the effect of the atmospheric refraction can be demonstrated by computing the height difference

$$
\Delta h_{A B}=\sqrt{R^{2}+s^{2}+2 R s \cos z_{A}}-R
$$

between the two points $A$ and $B$, that corresponds to the applied spherical approximation. If we evaluate equation (31) using for the zenith angle the two different values $z_{A}^{\prime}$ (spherical refraction model) and $z_{A}$ (absence of refraction, linear line propagation approximation) we obtain respectively

$$
\begin{aligned}
& \Delta h\left(\text { from } z_{A}^{\prime}\right)=785.095 \mathrm{~m} \\
& \Delta h\left(\text { from } z_{A}\right)=784.088 \mathrm{~m}
\end{aligned}
$$

which demonstrates that the magnitude of neglecting the effect of the atmospheric refraction leads in this case to a difference in the computed trigonometric height difference equal to 1.007 meters. 


\section{Exact algebraic solution}

An alternative way of evaluating the fundamental equation (12) is through its transformation to an algebraic equation of fourth order, with an appropriate choice of parameter substitutions. By introducing the following auxiliary variables

$$
\mu=\frac{\gamma}{2} \quad, \quad a=\frac{s}{r} \quad, \quad b=\frac{1}{2}\left(\pi+z_{A}^{\prime}-z_{B}^{\prime}\right)
$$

equation (12) becomes

$$
\sin 2 \mu=a \sin (b-\mu)
$$

or equivalently, after developing the respective trigonometric expressions

$$
2 \sin \mu \cos \mu=a \sin b \cos \mu-a \cos b \sin \mu
$$

At this point we define the following auxiliary variable

$$
t=\tan \frac{\mu}{2}
$$

which represents the fundamental unknown parameter of the subsequent exact algebraic solution. Based on this definition we obtain for the sinus and co-sinus of angle $\mu$ the respective expressions

$$
\cos \mu=\frac{1-t^{2}}{1+t^{2}} \quad, \quad \sin \mu=\frac{2 t}{1+t^{2}}
$$

Inserting the abbreviations

$$
c=a \sin b \quad ; \quad d=-a \cos b
$$

in equation (34) we obtain

$$
4 t\left(1-t^{2}\right)=c\left(1-t^{2}\right)\left(1+t^{2}\right)+d 2 t\left(1+t^{2}\right)
$$


and finally

$$
c t^{4}-(4+2 d) t^{3}+(4-2 d) t-c=0
$$

One way to solve the fourth degree equation (39) is described in Bronstein and Semendjajew (2002). According to this approach equation (39) can be transformed through an appropriate parameter substitution to the reduced equation $y^{4}+p y^{3}+q y+r=0$, whose solution depends on the so-called cubic resolvent $z^{3}+2 p z^{2}+\left(p^{2}-4 r\right) z-q^{2}=0$. After some further algebraical definitions and parameter prerequisites one obtains finally 4 independent solutions for $y$, from which the correct one has to be retained. Although the problem of solving (39) can be brought in algebraic exact form, the numerical implementation of this algebraic method is tedious and not simple to programm. Since the simple iterative method, proposed in the previous section is reliable and makes the computation of the exact solution with any desired accuracy possible, there is no actual need to use the algebraic method in practical applications.

\section{Approximate solution}

Applying certain approximations one may arrive to an alternative expression for parameters $\delta$ and $k$, that can be found in the geodetic literature. We will refer to Figure 2 and mainly to Figure 3 in order to examine this approximate solution. From Figure 2 it holds $2 \delta=s_{1} r$, where $s_{1}$ represents the spherical light propagation model that bends the horizontal light curve to an arc defined on the respective circle of radius $r$. Thus for angle $\delta$ we obtain

$$
\delta=\frac{s_{1}}{2 r}
$$

Moving on to Figure 3 we proceed with the definition of two additional quantities. We denote with $s_{H}$ the so-called horizontal distance, i.e. the distance separating $A$ from the plumb line at $B$, measured along the direction for which $z_{A}^{\prime}=100$ gon $\left(90^{\circ}\right)$, i.e. a direction defined on the horizontal plane at $A$. Performing the approximation $s_{1} \approx s \approx s_{H}$ we have

$$
\delta \approx \frac{s_{H}}{2 r}
$$

Inserting

$$
\frac{1}{r}=\frac{k}{R_{o}}
$$


from equation (2) into (41) we get

$$
\delta \approx \frac{s_{H} k}{2 R_{o}}
$$

Angle $\gamma$ on the other hand is defined from Figure 3 with respect to the dimensions of the "Earth sphere" as follows

$$
\gamma=\frac{S}{R}
$$

with $S$ the arc that is defined on the "Earth sphere". Using the additional approximation $s_{H} \approx S$ and $R \approx R_{o}$ we have for $\gamma$ the approximate representation

$$
\gamma \approx \frac{s_{H}}{R}
$$

In addition, from the equality

$$
z_{A}^{\prime}+\delta+z_{B}^{\prime}+\delta=\pi+\gamma
$$

it follows

$$
z_{A}^{\prime}+z_{B}^{\prime}+2 \delta=\pi+\gamma
$$

Combining equations (43), (45) and (47) the following approximation is obtained

$$
z_{A}^{\prime}+z_{B}^{\prime}+\frac{s_{H} k}{R} \approx \pi+\frac{s_{H}}{R}
$$

Solving this expression for $k$ we are finally led to the following approximate expression for the refraction coefficient (e.g., Kahmen and Faig 1988)

$$
k \approx 1-\left(z_{A}^{\prime}+z_{B}^{\prime}-\pi\right) \frac{R}{s_{H}}
$$

The respective angle correction $\delta$ to the zenith observations $z_{A}^{\prime}$ and $z_{B}^{\prime}$ is then computed by inserting (49) into (43) to obtain

$$
\delta \approx \frac{s_{H}-\left(z_{A}^{\prime}+z_{B}^{\prime}-\pi\right) R}{2 R}
$$


In the course of the derivation of relations (49) and (50) approximations were introduced in practically every step. Hence, it is not easy to estimate theoretically how significant are the errors induced in this way. However, the approximate expression (49) can be assessed numerically with the help of synthetic data. In the data generation process one must be cautious not to generate data using for example the approximate expression (49). Hence, some of the parameters should be chosen according to the domain of values which might be of interest in practical applications, while the rest will be computed using the appropriate formulae. For the subsequent numerical investigations we chose $R, k, \gamma$ and $z_{A}$ as the arbitrarily defined parameters, whereas all other involved quantities $\left(S, s, s_{H}, z_{B}, \delta\right)$ are obtained from the respective exact relations valid in the frame of the postulated spherical model. In more detail, equation (2) produces directly the value for parameter $r$. For the arc length $S$ the standard formula $S=R \gamma$ is applied. The slope distance $s$ and the horizontal distance $s_{H}$ can be computed by means of the expressions (7) and $s_{H}=R \tan \gamma$ respectively. Furthermore the opposite zenith angle $z_{B}$ follows from the obvious expression $z_{B}=\pi+\gamma-z_{A}$ and finally the angle $\delta$ can be obtained by simply evaluating the formula

$$
\delta=\arcsin \left(\frac{s}{2 r}\right)
$$

The computations which are presented in Tables $1-4$ refer to a different postulated $k$ values. These values were selected from the realistic range -0.13 to 0.52 with an interval of 0.13 and correspond to the 'true' refraction coefficient that holds at every synthetic computation. In other words for the results presented in each of the Tables 1-4 we assume that the real value of $k$ is the one used in the data generation process and we investigate the deviations of the computed values obtained from the approximation formula (49) in order to investigate its performance in different scenarios. The presented numerical values denote the computed $k$ values as obtained from the numerical implementation of (49) (first row of each table entry) and the calculated error for the correction of the zenith angle observations, i.e. the difference between the computed (Eq. (50)) and the expected numerical value of angle $\delta$ (Eq. (51)) expressed in mgon (second row of each table entry). The computations were implemented for different zenith angles and slope distances, ranging from 50 gon $\left(45^{\circ}\right)$ to 150 gon $\left(135^{\circ}\right)$ and from $500.30 \mathrm{~m}$ to $16009.59 \mathrm{~m}$ respectively. Empty table entries express combinations of zenith angle and slope distance data that cannot correspond to real-case experiments, in other words to arrangements that are hardly or not at all realizable in real field cases.

Tables 1-4 offer an interesting insight into the accuracy of the standard approximate solution for $k$. It becomes evident, that the validity of equation (49) depends strongly on the basic prerequisite of the approach that $s_{1} \approx s \approx s_{H} \approx S$. This is demonstrated clearly by the produced results. The approximate solution produces zero deviations from the 'true' $k$-values for zenith angle observations equal to 100 gon $\left(90^{\circ}\right)$, i.e. in those cases where the slope distance coincides with the horizontal distance. In the 
surrounding neighborhood of this value the approximate method continues to produce acceptable results, with differences both in $k$ and $\delta$ which are so small that they can be regarded as negligible. In principle, one may use as a measure for the obtained errors in the computation of the angle correction $\delta$, by comparing them with the overall reading and/or angle measurement precision, which commonly does not exceed the 0.1 mgon $\left(0.32^{\prime \prime}\right)$ level. The regions where this numerical threshold is valid are marked with boldface characters. The results demonstrate that the width of this region diminishes with increasing distance between the two field points, while it also decreases with increasing (in absolute sense) value of $k$. Outside these limits the approximate solution for the atmospheric refraction coefficient produces results that deviate up to $40 \%$ compared to the predefined 'true' $k$-values. The deviations of the computed from the expected $\delta$ values seem to vary proportionally with the slope distance $s$ and the refraction coefficient $k$, since they depend directly on the zenith angle observation. Concluding, for all considered $k$ values, the approximate formula for the determination of the refraction coefficient $k$ is valid only for horizontal sightings, or sightings that are very close to the horizontal plane that is defined at $A$. However, this corresponds to only a limited real-case scenario, and prohibits the application of the mutual zenith angle observation setting to the most interesting case of a non negligible real height difference between the points $A$ and $B$. Furthermore, our numerical investigations showed, that the approximate solution (49) offers no advantages (neither theoretical nor numerical) compared to the iterative solution presented in section 3. There are no mathematical tricks which can replace missing information. However, even under stable atmospheric conditions, when the simple spherical symmetric model represents well the physical reality, the traditional approximate solution introduces unnecessary errors which limit the applicability considerably. In the age of logarithmic and trigonometric tables and less precise instruments such a procedure made sense, however since the invention of pocket calculators it became rather meaningless. Thus, the simple and efficient iterative solution proposed in this paper should be clearly preferred over all other variants in practical applications.

\section{Concluding remarks}

The knowledge of a detailed model describing the effect of atmospheric refraction to field geodetic measurements is of crucial importance for many standard geodetic applications. Especially in rough terrain and large real height differences combined with increased slope distances between observed and observation point, the correction of the effect of atmospheric refraction in geometric and trigonometric leveling is important in order to obtain the standard accuracy. The present discussion revisited the problem of atmospheric refraction coefficient determination using the field setup of simultaneously registered mutual zenith observations. The approximate solution for the spherical symmetric model, met in standard geodetic references, is valid only under a series of subsequent and sometimes rightfully questionable assumptions. In other words, even under stable atmospheric conditions, when the simple spherical sym- 
metric model represents well the physical reality, the approximate solution introduces unnecessary errors which limit the domain of its applicability considerably. If no other information or measurement facility is available, the iterative solution proposed in this paper offers an exact and rigorous representation of the spherical symmetric refraction model, that can be easily adopted to produce directly preprocessed trigonometric and geometric leveling data, providing that some couples of mutual zenith observations are available, with varying slope distances and chosen appropriately within the limits of the area in question. This iterative computation produces a solution with a sufficient precision within only a couple of iterations for most practical cases and can be performed on any pocket calculator. 


\section{References}

Bronstein, I.N., and Semendjajew, K.A. (2002). Handbook of Mathematics, Springer, Heidelberg.

Cohen, I.B. (1980). From Leonardo to Lavoisier 1450-1800 (Album of Science), Charles Scribner's Sons, New York.

Flach, P., and Maas, H.G. (1999). "Vision-based Techniques for Refraction Analysis in Applications of Terrestrial Geodesy." Proc., ISPRS Workshop Vision-Based Techniques in Visualization and Animation, IAPRS Vol. XXXII-5/W13, The Netherlands, 195-201.

Gottwald, R. (1985). "Zur Genauigkeitssteigerung und Erstellung eines automatisierten Datenflusses beim trigonometrischen Nivellement mit kurzen Zielweiten." Ph.D. thesis, Veröffentlichung des Geodätischen Instituts der Rheinisch-Westfälischen Technischen Hochschule Aachen, Nr. 37, Aachen.

Hennes, M. (2002). "Zum Refraktionseinfluss auf terrestrische geodatische Messungen im Kontext der Messtechnik und der Instrumentenentwicklung." Flachenmanagement und Bodenneuordnung (FuB), 2, 73-86.

Jordan, W., Eggert, O., and Kneissl, M. (1956). Handbuch der Vermessungskunde, Band III (Hohenmessung, Tachymetrie), JB Metzlersche Verlagsbuchhandlung, Stuttgart.

Kahmen, H., and Faig, W. (1988). Surveying, Walter de Gruyter, Berlin-New York.

Murdoch, J.E. (1984). Antiquity and the Middle Ages (Album of Science), Charles Scribner's Sons, New York.

Rüeger, J.M. (1996). Electronic Distance Measurement - An Introduction, 4th Ed., Springer-Verlag, BerlinHeidelberg-New York.

Witte, B., and Binnenbruck, B. (2000). "Present and future ways of determining the effect of vertical refraction by use of digital surveying instruments." Proc., FIG Seminar Mediterranean Surveyor in the new Millenium (CD-ROM), FIG, Denmark. 


\section{Tables}

Table 1. Numerical assessment of the approximation formulae (49)-(50) with the postulated value for the refraction coefficient $k=-0.13$. The first row values present the computed refraction coefficient while the second and third rows give the difference between computed (50) and expected (51) value for quantity $\delta$ expressed in mgon and arcsec respectively.

\begin{tabular}{|c|c|c|c|c|c|c|c|}
\hline \multirow[b]{2}{*}{$\begin{array}{c}\text { Zenith angle } \\
\text { in degrees }\end{array}$} & \multicolumn{6}{|c|}{ Slope distance $(A B)$ in meters } & \multirow[b]{2}{*}{16009.59} \\
\hline & $\begin{array}{c}\text { Zenith angle } \\
\text { in gon }\end{array}$ & 500.30 & 1000.60 & 2001.19 & 4002.39 & 8004.78 & \\
\hline \multirow[t]{3}{*}{45} & 50 & -0.184 & -0.184 & -0.184 & & & \\
\hline & & -0.19 & -0.38 & -0.76 & & & \\
\hline & & -0.61 & -1.23 & -2.46 & & & \\
\hline \multirow[t]{3}{*}{54} & 60 & -0.161 & -0.161 & -0.161 & -0.161 & & \\
\hline & & -0.09 & -0.19 & -0.38 & -0.76 & & \\
\hline & & -0.29 & -0.61 & -1.23 & -2.46 & & \\
\hline \multirow[t]{3}{*}{63} & 70 & -0.146 & -0.146 & -0.146 & -0.146 & & \\
\hline & & -0.04 & -0.09 & -0.18 & -0.36 & & \\
\hline & & -0.12 & -0.29 & -0.58 & -1.16 & & \\
\hline \multirow[t]{3}{*}{72} & 80 & -0.137 & -0.137 & -0.137 & -0.137 & -0.137 & \\
\hline & & -0.02 & -0.04 & -0.07 & -0.14 & -0.28 & \\
\hline & & -0.06 & -0.12 & -0.22 & -0.45 & -0.91 & \\
\hline \multirow[t]{3}{*}{81} & 90 & -0.132 & -0.132 & -0.132 & -0.132 & -0.132 & -0.132 \\
\hline & & 0.00 & -0.01 & -0.02 & -0.03 & -0.07 & -0.14 \\
\hline & & 0.00 & -0.03 & -0.06 & -0.09 & -0.07 & -0.45 \\
\hline \multirow[t]{3}{*}{90} & 100 & -0.130 & -0.130 & -0.130 & -0.130 & -0.130 & -0.130 \\
\hline & & 0.00 & 0.00 & 0.00 & 0.00 & 0.00 & 0.00 \\
\hline & & 0.00 & 0.00 & 0.00 & 0.00 & 0.00 & 0.00 \\
\hline \multirow[t]{3}{*}{99} & 110 & -0.132 & -0.132 & -0.132 & -0.132 & -0.132 & -0.132 \\
\hline & & 0.00 & -0.01 & -0.02 & -0.03 & -0.06 & -0.13 \\
\hline & & 0.00 & -0.03 & -0.06 & -0.09 & -0.19 & -0.42 \\
\hline \multirow[t]{3}{*}{108} & 120 & -0.137 & -0.137 & -0.137 & -0.137 & -0.137 & \\
\hline & & -0.02 & -0.04 & -0.07 & -0.14 & -0.28 & \\
\hline & & -0.06 & -0.12 & -0.22 & -0.45 & -0.91 & \\
\hline \multirow[t]{3}{*}{117} & 130 & -0.146 & -0.146 & -0.146 & -0.146 & & \\
\hline & & -0.04 & -0.09 & -0.18 & -0.36 & & \\
\hline & & -0.12 & -0.29 & -0.58 & -1.16 & & \\
\hline \multirow[t]{3}{*}{126} & 140 & -0.161 & -0.161 & -0.161 & -0.161 & & \\
\hline & & -0.09 & -0.19 & -0.38 & -0.76 & & \\
\hline & & -0.29 & -0.61 & -1.23 & -2.46 & & \\
\hline \multirow[t]{3}{*}{135} & 150 & -0.184 & -0.184 & -0.184 & & & \\
\hline & & -0.19 & -0.38 & -0.76 & & & \\
\hline & & -0.61 & -1.23 & -2.46 & & & \\
\hline
\end{tabular}


Table 2. Numerical assessment of the approximation formulae (49)-(50) with the postulated value for the refraction coefficient $k=0.26$. The first row values present the computed refraction coefficient while the second and third rows give the difference between computed (50) and expected (51) value for quantity $\delta$ expressed in mgon and arcsec respectively.

\begin{tabular}{|c|c|c|c|c|c|c|c|}
\hline \multirow[b]{2}{*}{$\begin{array}{c}\text { Zenith angle } \\
\text { in degrees }\end{array}$} & \multicolumn{6}{|c|}{ Slope distance $(A B)$ in meters } & \multirow[b]{2}{*}{16009.59} \\
\hline & $\begin{array}{l}\text { Zenith angle } \\
\text { in gon }\end{array}$ & 500.30 & 1000.60 & 2001.19 & 4002.39 & 8004.78 & \\
\hline \multirow[t]{3}{*}{45} & 50 & 0.368 & 0.368 & 0.368 & & & \\
\hline & & 0.38 & 0.76 & 1.53 & & & \\
\hline & & 1.23 & 2.46 & 4.96 & & & \\
\hline \multirow[t]{3}{*}{54} & 60 & 0.321 & 0.321 & 0.321 & 0.322 & & \\
\hline & & 0.19 & 0.38 & 0.76 & 1.52 & & \\
\hline & & 0.61 & 1.23 & 2.46 & 4.96 & & \\
\hline \multirow[t]{3}{*}{63} & 70 & 0.292 & 0.292 & 0.292 & 0.292 & & \\
\hline & & 0.09 & 0.18 & 0.36 & 0.72 & & \\
\hline & & 0.29 & 0.58 & 1.23 & 2.46 & & \\
\hline \multirow[t]{3}{*}{72} & 80 & 0.273 & 0.273 & 0.273 & 0.273 & 0.273 & \\
\hline & & 0.04 & 0.07 & 0.14 & 0.28 & 0.57 & \\
\hline & & 0.12 & 0.22 & 0.45 & 0.91 & 1.85 & \\
\hline \multirow[t]{3}{*}{81} & 90 & 0.263 & 0.263 & 0.263 & 0.263 & 0.263 & 0.263 \\
\hline & & 0.01 & 0.02 & 0.03 & 0.07 & 0.13 & 0.27 \\
\hline & & 0.03 & 0.06 & 0.09 & 0.22 & 0.42 & 0.87 \\
\hline \multirow[t]{3}{*}{90} & 100 & 0.260 & 0.260 & 0.260 & 0.260 & 0.260 & 0.260 \\
\hline & & 0.00 & 0.00 & 0.00 & 0.00 & 0.00 & 0.00 \\
\hline & & 0.00 & 0.00 & 0.00 & 0.00 & 0.00 & 0.00 \\
\hline \multirow[t]{3}{*}{99} & 110 & 0.263 & 0.263 & 0.263 & 0.263 & 0.263 & 0.263 \\
\hline & & 0.01 & 0.02 & 0.03 & 0.07 & 0.13 & 0.25 \\
\hline & & 0.03 & 0.06 & 0.09 & 0.22 & 0.42 & 0.81 \\
\hline \multirow[t]{3}{*}{108} & 120 & 0.273 & 0.273 & 0.273 & 0.273 & 0.273 & \\
\hline & & 0.04 & 0.07 & 0.14 & 0.28 & 0.56 & \\
\hline & & 0.12 & 0.22 & 0.45 & 0.91 & 1.81 & \\
\hline \multirow[t]{3}{*}{117} & 130 & 0.292 & 0.292 & 0.292 & 0.292 & & \\
\hline & & 0.09 & 0.18 & 0.36 & 0.71 & & \\
\hline & & 0.29 & 0.58 & 1.23 & 2.30 & & \\
\hline \multirow[t]{3}{*}{126} & 140 & 0.321 & 0.321 & 0.321 & 0.322 & & \\
\hline & & 0.19 & 0.38 & 0.76 & 1.51 & & \\
\hline & & 0.61 & 1.23 & 2.46 & 4.89 & & \\
\hline \multirow[t]{3}{*}{135} & 150 & 0.368 & 0.368 & 0.368 & & & \\
\hline & & 0.38 & 0.76 & 1.52 & & & \\
\hline & & 1.23 & 2.46 & 4.96 & & & \\
\hline
\end{tabular}


Table 3. Numerical assessment of the approximation formulae (49)-(50) with the postulated value for the refraction coefficient $k=0.39$. The first row values present the computed refraction coefficient while the second and third rows give the difference between computed (50) and expected (51) value for quantity $\delta$ expressed in mgon and arcsec respectively.

\begin{tabular}{|c|c|c|c|c|c|c|c|}
\hline \multirow[b]{2}{*}{$\begin{array}{c}\text { Zenith angle } \\
\text { in degrees }\end{array}$} & \multicolumn{6}{|c|}{ Slope distance $(A B)$ in meters } & \multirow[b]{2}{*}{16009.59} \\
\hline & $\begin{array}{l}\text { Zenith angle } \\
\text { in gon }\end{array}$ & 500.30 & 1000.60 & 2001.19 & 4002.39 & 8004.78 & \\
\hline \multirow[t]{3}{*}{45} & 50 & 0.552 & 0.552 & 0.552 & & & \\
\hline & & 0.57 & 1.14 & 2.29 & & & \\
\hline & & 1.85 & 3.69 & 7.42 & & & \\
\hline \multirow[t]{3}{*}{54} & 60 & 0.482 & 0.482 & 0.482 & 0.482 & & \\
\hline & & 0.28 & 0.57 & 1.14 & 2.28 & & \\
\hline & & 0.91 & 1.85 & 3.69 & 7.39 & & \\
\hline \multirow[t]{3}{*}{63} & 70 & 0.438 & 0.438 & 0.438 & 0.438 & & \\
\hline & & 0.13 & 0.27 & 0.54 & 1.07 & & \\
\hline & & 0.42 & 0.87 & 1.75 & 3.47 & & \\
\hline \multirow[t]{3}{*}{72} & 80 & 0.410 & 0.410 & 0.410 & 0.410 & 0.410 & \\
\hline & & 0.05 & 0.11 & 0.21 & 0.42 & 0.85 & \\
\hline & & 0.16 & 0.36 & 0.68 & 1.36 & 2.75 & \\
\hline \multirow[t]{3}{*}{81} & 90 & 0.395 & 0.395 & 0.395 & 0.395 & 0.395 & 0.395 \\
\hline & & 0.01 & 0.02 & 0.05 & 0.10 & 0.20 & 0.41 \\
\hline & & 0.03 & 0.06 & 0.16 & 0.32 & 0.65 & 1.33 \\
\hline \multirow[t]{3}{*}{90} & 100 & 0.390 & 0.390 & 0.390 & 0.390 & 0.390 & 0.390 \\
\hline & & 0.00 & 0.00 & 0.00 & 0.00 & 0.00 & 0.00 \\
\hline & & 0.00 & 0.00 & 0.00 & 0.00 & 0.00 & 0.00 \\
\hline \multirow[t]{3}{*}{99} & 110 & 0.395 & 0.395 & 0.395 & 0.395 & 0.395 & 0.395 \\
\hline & & 0.01 & 0.02 & 0.05 & 0.10 & 0.19 & 0.38 \\
\hline & & 0.03 & 0.06 & 0.16 & 0.32 & 0.61 & 1.23 \\
\hline \multirow[t]{3}{*}{108} & 120 & 0.410 & 0.410 & 0.410 & 0.410 & 0.410 & \\
\hline & & 0.05 & 0.11 & 0.21 & 0.42 & 0.84 & \\
\hline & & 0.16 & 0.36 & 0.68 & 1.36 & 2.72 & \\
\hline \multirow[t]{3}{*}{117} & 130 & 0.438 & 0.438 & 0.438 & 0.438 & & \\
\hline & & 0.13 & 0.27 & 0.53 & 1.07 & & \\
\hline & & 0.42 & 0.87 & 1.72 & 3.47 & & \\
\hline \multirow[t]{3}{*}{126} & 140 & 0.482 & 0.482 & 0.482 & 0.482 & & \\
\hline & & 0.28 & 0.57 & 1.14 & 2.27 & & \\
\hline & & 0.91 & 1.85 & 3.69 & 7.35 & & \\
\hline \multirow[t]{3}{*}{135} & 150 & 0.552 & 0.552 & 0.552 & & & \\
\hline & & 0.57 & 1.14 & 2.28 & & & \\
\hline & & 1.85 & 3.69 & 7.39 & & & \\
\hline
\end{tabular}


Table 4. Numerical assessment of the approximation formulae (49)-(50) with the postulated value for the refraction coefficient $k=0.52$. The first row values present the computed refraction coefficient while the second and third rows give the difference between computed (50) and expected (51) value for quantity $\delta$ expressed in mgon and arcsec respectively.

\begin{tabular}{|c|c|c|c|c|c|c|c|}
\hline \multirow[b]{2}{*}{$\begin{array}{c}\text { Zenith angle } \\
\text { in degrees }\end{array}$} & \multicolumn{6}{|c|}{ Slope distance $(A B)$ in meters } & \multirow[b]{2}{*}{16009.59} \\
\hline & $\begin{array}{l}\text { Zenith angle } \\
\text { in gon }\end{array}$ & 500.30 & 1000.60 & 2001.19 & 4002.39 & 8004.78 & \\
\hline \multirow[t]{3}{*}{45} & 50 & 0.735 & 0.736 & 0.736 & & & \\
\hline & & 0.76 & 1.52 & 3.05 & & & \\
\hline & & 2.46 & 4.96 & 9.88 & & & \\
\hline \multirow[t]{3}{*}{54} & 60 & 0.643 & 0.643 & 0.643 & 0.643 & & \\
\hline & & 0.38 & 0.76 & 1.52 & 3.04 & & \\
\hline & & 1.23 & 2.46 & 4.96 & 9.85 & & \\
\hline \multirow[t]{3}{*}{63} & 70 & 0.584 & 0.584 & 0.584 & 0.584 & & \\
\hline & & 0.18 & 0.36 & 0.72 & 1.43 & & \\
\hline & & 0.58 & 1.16 & 2.46 & 4.63 & & \\
\hline \multirow[t]{3}{*}{72} & 80 & 0.547 & 0.547 & 0.547 & 0.547 & 0.547 & \\
\hline & & 0.07 & 0.14 & 0.28 & 0.57 & 1.14 & \\
\hline & & 0.22 & 0.45 & 0.91 & 1.85 & 3.69 & \\
\hline \multirow[t]{3}{*}{81} & 90 & 0.526 & 0.526 & 0.527 & 0.527 & 0.527 & 0.527 \\
\hline & & 0.02 & 0.03 & 0.07 & 0.13 & 0.27 & 0.54 \\
\hline & & 0.06 & 0.09 & 0.22 & 0.42 & 0.87 & 1.75 \\
\hline \multirow[t]{3}{*}{90} & 100 & 0.520 & 0.520 & 0.520 & 0.520 & 0.520 & 0.520 \\
\hline & & 0.00 & 0.00 & 0.00 & 0.00 & 0.00 & 0.00 \\
\hline & & 0.00 & 0.00 & 0.00 & 0.00 & 0.00 & 0.00 \\
\hline \multirow[t]{3}{*}{99} & 110 & 0.526 & 0.526 & 0.526 & 0.526 & 0.526 & 0.526 \\
\hline & & 0.02 & 0.03 & 0.07 & 0.13 & 0.26 & 0.51 \\
\hline & & 0.06 & 0.09 & 0.22 & 0.42 & 0.84 & 1.65 \\
\hline \multirow[t]{3}{*}{108} & 120 & 0.547 & 0.547 & 0.547 & 0.547 & 0.547 & \\
\hline & & 0.07 & 0.14 & 0.28 & 0.56 & 1.12 & \\
\hline & & 0.22 & 0.45 & 0.91 & 1.81 & 3.63 & \\
\hline \multirow[t]{3}{*}{117} & 130 & 0.584 & 0.584 & 0.584 & 0.583 & & \\
\hline & & 0.18 & 0.36 & 0.71 & 1.42 & & \\
\hline & & 0.58 & 1.16 & 2.30 & 4.60 & & \\
\hline \multirow[t]{3}{*}{126} & 140 & 0.643 & 0.643 & 0.643 & 0.642 & & \\
\hline & & 0.38 & 0.76 & 1.52 & 3.03 & & \\
\hline & & 1.23 & 2.46 & 4.96 & 9.82 & & \\
\hline \multirow[t]{3}{*}{135} & 150 & 0.735 & 0.735 & 0.735 & & & \\
\hline & & 0.76 & 1.52 & 3.04 & & & \\
\hline & & 2.46 & 4.96 & 9.85 & & & \\
\hline
\end{tabular}




\section{Figure Captions}

Fig. 1. Basic geometric definitions for the simultaneous and mutual zenith observations setup.

Fig. 2. The model assumes a spherical approximation for the actual optical ray path.

Fig. 3. Geometric quantities linked to the approximate solution for the refraction coefficient determination from the mutual zenith observation setup. 


\section{Figures}

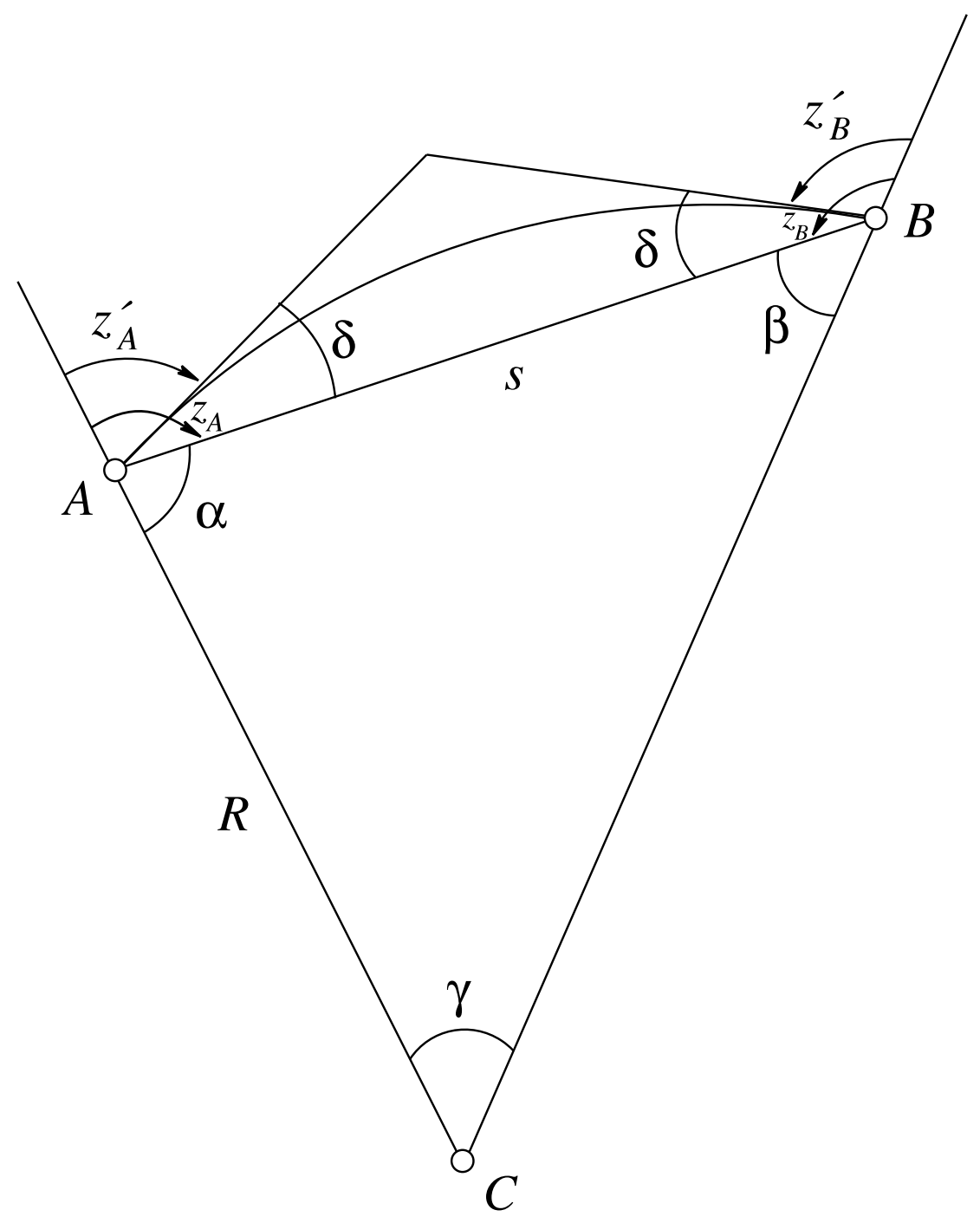

Fig. 1. Basic geometric definitions for the simultaneous and mutual zenith observations setup. 


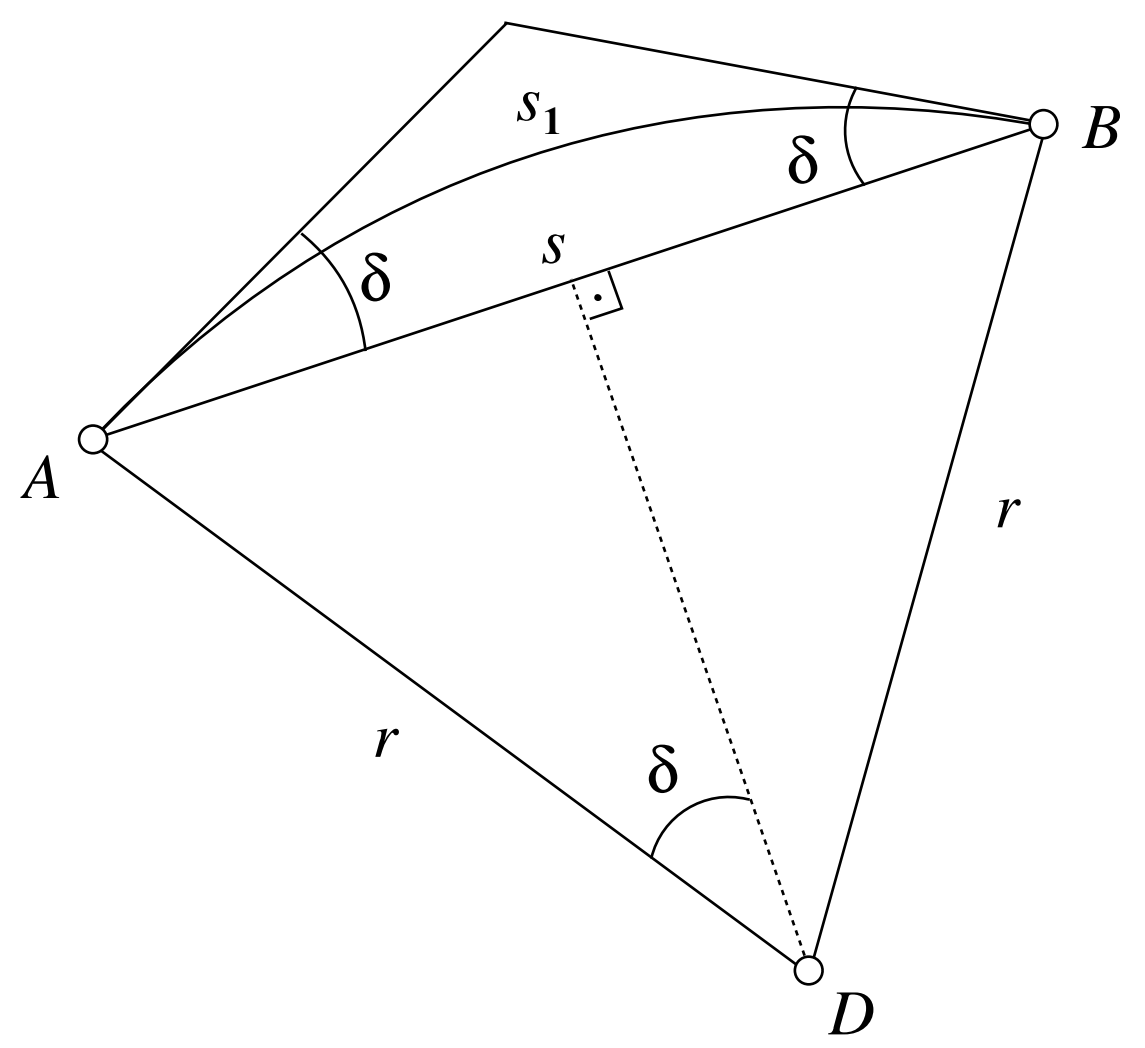

Fig. 2. The model assumes a spherical approximation for the actual optical ray path. 


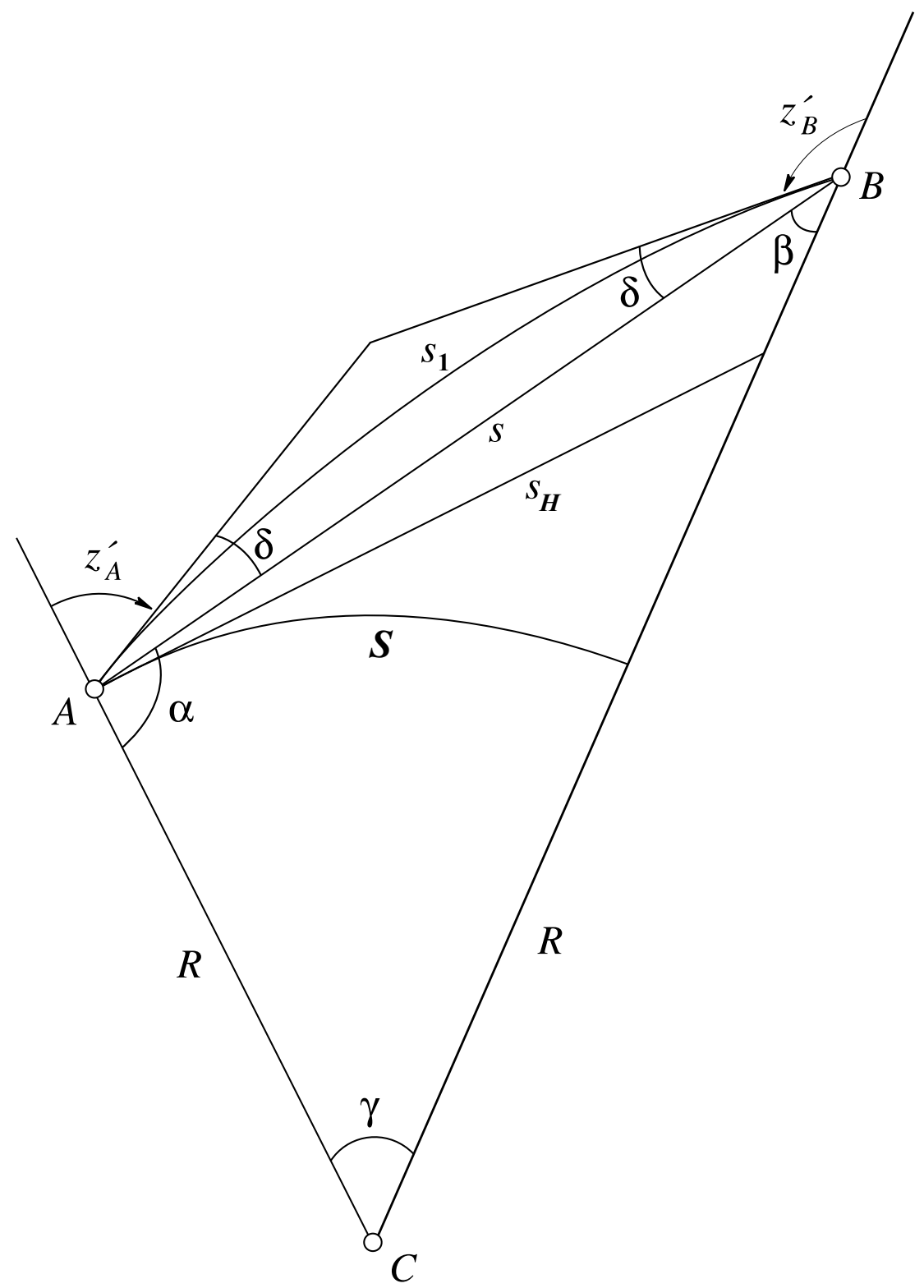

Fig. 3. Geometric quantities linked to the approximate solution for the refraction coefficient determination from the mutual zenith observation setup. 